\title{
Progress in Electrons Vortex Creation and Application in a Transmission Electron Microscope
}

\author{
J. Verbeeck ${ }^{1}$, A. Béché ${ }^{1}$, L. Clark ${ }^{1}$, G. Guzzinati ${ }^{1}$, R. Juchtmans ${ }^{1}$, A. Lubk ${ }^{2}$, H. Tian ${ }^{1}$, R. Van Boxem ${ }^{1}$, \\ G. Van Tendeloo ${ }^{1}$ \\ 1. EMAT, University of Antwerp, Antwerp, Belgium \\ 2. Triebenberlabor, University of Dresden, Dresden, Germany
}

Vortex electron waves are the electron wave cousin of the better known optical vortices. In optics, they are attracting considerable interest both from a fundamental and application point of view. The essence of wave vortices is the presence of a phase singularity on the propagation axis leading to an extrinsic orbital angular momentum (OAM) in contrast to the intrinsic spin angular momentum (SAM) carried by the polarisation of the wave [1,2]. A typical way to produce such a vortex wave would be a phase plate with spiraling thickness profile leading to an integer times $2 \pi$ phase shift when going around the optical axis as sketched in Fig.1. This integer is called the topological charge $l$ which links to the orbital angular momentum of $l \mathrm{~h}$ per elementary particle in the wave.

Applying these concepts to electron waves as available in a transmission electron microscope, one can indeed produce electron vortices e.g. making use of a holographic reconstruction technique $[3,4]$. Holographic masks containing either a fork or a spiral structure were shown to produce vortex waves with different topological charge separated in space [4-6]. The size of such a vortex wave was shown to reach the atomic scale [5-6] as was proven from a direct measurement of an image of the probe as well as from making use of vortex probes in scanning transmission electron microscopy (STEM) imaging while still retaining atomic resolution. Many different approaches exist to obtain electron vortices in a transmission electron microscope and we will discuss their working principle and experimental implementation. A recent example is given in Fig.2 where we use the control over the aberration function of a modern probe corrected electron microscope in combination with an annular condenser aperture to obtain an approximation of a vortex beam [7]. The advantage of this approach is that a single vortex probe is generated as opposed to the unwanted sidebands created with holographic reconstruction techniques. Alternative techniques based on the interaction of electrons with a localized magnetic field will be demonstrated as well. This technical evolution in the creation of single electron vortices at high current and of atomic size is of major importance for applications.

Apart from creating vortex beams, we also discuss the creation of an analyzer for the OAM of an existing wave. Also here, many different implementations exist e.g. using fork apertures or a method based on the optical Gouy phase [8,9].

In terms of applications, we will demonstrate advances in local magnetic measurements via electron energy loss spectroscopy as well as nanomanipulation experiments [10]. These experimental results point out the importance of a better theoretical understanding of the interaction of electron vortices with matter.

Recent progress on how topological and symmetry arguments are involved in the elastic scattering of vortex electrons will be presented [11]. We will show that electron vortices are virtually omnipresent when scattering with matter as the creation and annihilation of vortex-antivortex pairs is a common feature in any TEM experiment. The subtle distinction between the spin and orbital part of the angular momentum is clarified making use of a relativistic quantum treatment [12]. Adding inelastic scattering complicates the interaction further but numerical simulations show that atomic resolution magnetic mapping remains feasible for thin samples [13]. 
[1] J F Nye and M V Berry, Proceedings of the Royal Society A 336 (1974) p. 165.

[2] L Allen et al., Physical review. A 45 (1992) p. 8185.

[3] M Uchida and A Tonomura, Nature 464 (2010) p. 737.

[4] J Verbeeck et al., Nature 467 (2010) p. 301.

[5] J. Verbeeck, et al., Ultramicroscopy 113 (2012) p. 83.

[6] J. Verbeeck et al., Applied Physics Letters, vol. 99, no. 20, p. $203109,2011$.

[7] L. Clark et al., Physical Review Letters (2013) in preparation.

[8] K. Bliokh, et al., Physical Review X, 2, (2012) p. 041011.

[9] G. Guzzinati et al.,Physical Review Letters (2013) accepted.

[10] J. Verbeeck et al., Advanced Materials, (2012) in press. DOI: 10.1002/adma.201204206

[11] A. Lubk et al., Physical Review A (2013) submitted.

[12] R Van Boxem et al., Physical Review Letters (2013) submitted.

[13] The authors acknowledge funding from the European Research Council under the 7th Framework Program (FP7), ERC grant N²46791 - COUNTATOMS and ERC Starting Grant N²78510-

VORTEX. Financial support from the European Union under the 7th Framework Program under a contract for an Integrated Infrastructure Initiative $N^{\circ} 312483-$ ESTEEM2 is also acknowledged.

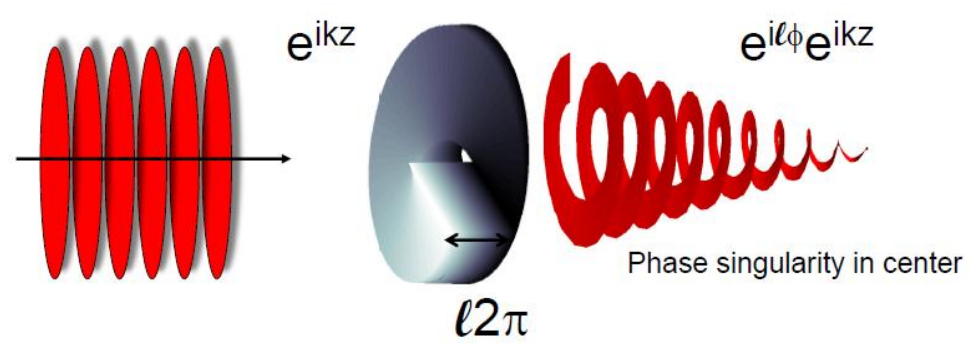

Figure 1. Schematic of the creation of a vortex wave with a spiral phase plate. Note how the wavefronts become helically connected and a phase singularity appears on the propagation axis.
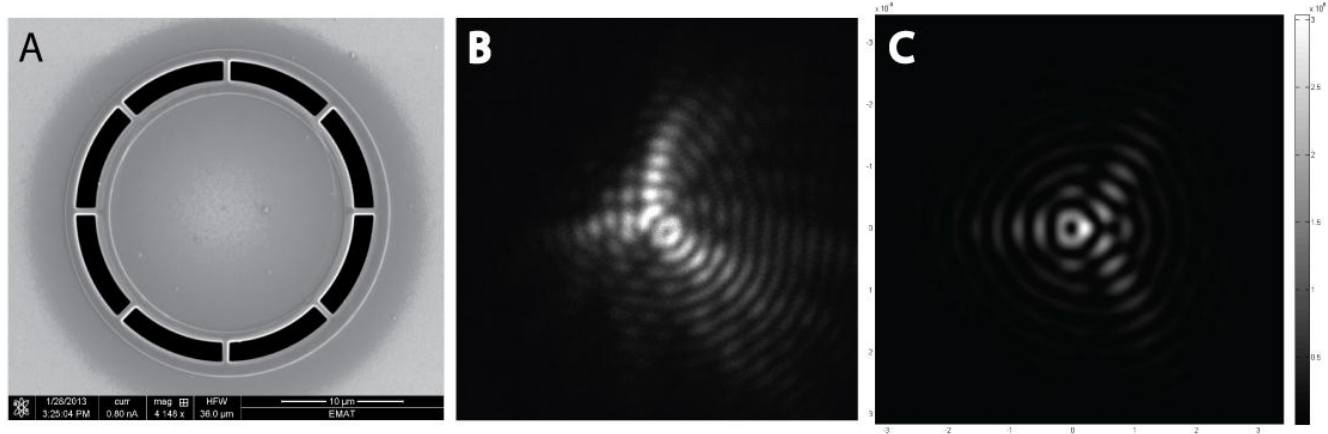

Figure 2. With the use of an annular condensor aperture (A) and controlled aberration tuning in a modern aberration corrected STEM instrument, a vortex electron beam can be created which is predominantly an $l=1$ vortex as seen in (B). The interference features are in close agreement to theoretical wave optical simulations with a ring like feature surrounding the vortex core (C). This process is yet another way to create vortex modes in a transmission electron microscope with the advantage of creating a single probe with vortex character. 